\title{
Numerical Simulation of DC Positive Air Corona Discharge under Atmospheric Environment
}

\author{
Xinghua Liu ${ }^{1,2, ~ a, ~ R i c h a n g ~ X i a n ~}{ }^{2, b, ~}{ }^{*}$, Yunzhu An ${ }^{2, c}$, Yuanchao $\mathrm{Hu}^{2, \mathrm{~d}}$, Yuyao $\mathrm{Hu}^{2, \mathrm{e}}$, Yang $\mathbf{Y u}^{1, \mathrm{f}}$, \\ and Ning Zhang ${ }^{2, g}$ \\ ${ }^{1}$ State Grid Zibo Power Supply Company, Shandong Electric Power Corporation, Shandong, 255000, China \\ ${ }^{2}$ School of Electrical and Electronic Engineering, Shandong University of Technology, Shandong 255000, \\ China \\ a30280241@qq.com, bxianrc@163.com, canyunzhu2006@163.com, dhuyuanchao3211@126.com, 'hyuyaocq

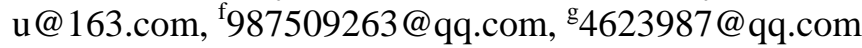

Keywords: DC positive, air corona discharge, atmospheric environment.

\begin{abstract}
This paper presents an improved self-consistent, multi-component and two-dimensional plasma hybrid model for the simulation of DC positive corona discharge under atmospheric environment. The model is based on the plasma hydrodynamics and the chemical models. It includes 12 species and 27 reactions. Besides, the photoionization effect is also considered in the proposed model. Based on this model, the volume of dynamics charged species generation, the discharge current waveform, electron temperature, and electric field variations are investigated in details. The results indicate that the model provides valuable insights to the physics of air corona discharge.
\end{abstract}

\section{Introduction}

Corona discharges are usually generated on sharp points, edges or on thin wires where the electric field is strongly concentrated. With the rapid development of extra and ultra high-voltage transmission lines, the air corona discharge becomes one of the critical problems associated with high-voltage lines, which would lead to the deterioration of insulation systems, power loss, radio noise. They have received the attention of many researchers.

Despite the recent improvements, the physical mechanism associated with the dynamics of corona discharge is still unclear. A effective tool to investigate the corona discharge in details is the introduction of numerical modeling. Nahomy [1] who adopted 430 chemistry reactions in air discharge, presented one of the most comprehensive compilations of chemistry mechanism. The mathematical modeling of DC or impulse discharge was utilized to explore the physical mechanism, and theories of the chemical reactions during the discharge process tend to be clear gradually after the above milestone paper. Macheret et al. [2] demonstrated the air plasma generation through repetitive high-voltage nanosecond pulses. Pancheshnyi et al. [3] developed a reduced air model with 10 species based on the mechanism of Nahomy [1] to simulate the streamer discharges at the pressure of 1 atm. Morrow [4] investigated the corona discharge in oxygen and other gases using the finite-element method. Gordiets [5] developed a reduced air chemistry model to investigate the role of excited neutral molecules and atoms in the air discharge. Recently, Liang et al. investigated the influence of reduced electric field on the evolvement characteristics of plasma for $\mathrm{N}_{2} / \mathrm{O}_{2}$ discharge. Yin et al. simulated the reaction diffusion process of air discharge in the open atmospheric environment.

In this paper, a self-consistent, multi-component and two-dimensional discharge hybrid model is developed to simulate the atmospheric environment DC positive corona discharge, which is based on the discharge hydrodynamics and the chemical model. And the photoionization effect is also considered. The aim of this work is to report the details of the developed discharge hybrid model, and try to understand the electrical and the physical characteristics of DC positive corona discharge in air. 


\section{Mathematical Model}

\subsection{Governing Equations}

Air discharge is described as a continuum media, consisting of coupled electron continuity equations, heavy species multi-component diffusion transport equations, momentum balance equations, electron energy balance equation and Poisson's equation for electric potential. Using the obtained electron energy distribution function as a solution of the Boltzmann equation, the electron mobility, diffusion coefficient and collision rate coefficients were calculated.

\subsection{Air Chemistry and Transport}

The air discharge model has 12 species: e, $\mathrm{O}, \mathrm{O}_{2}, \mathrm{O}_{3}, \mathrm{~N}_{2}{ }^{+}, \mathrm{N}_{4}{ }^{+}, \mathrm{O}_{2}{ }^{+}, \mathrm{O}_{4}{ }^{+}, \mathrm{N}_{2} \mathrm{O}_{2}{ }^{+}, \mathrm{O}_{2}{ }^{-}, \mathrm{O}^{-}$, and $\mathrm{N}_{2}$. A total of 27 reactions were considered, including elastic scattering, ionization, two-body recombination, three-body recombination, dissociative recombination, molecular ion conversion, excitation, de-excitation, and so on.

\subsection{Computational Implementation}

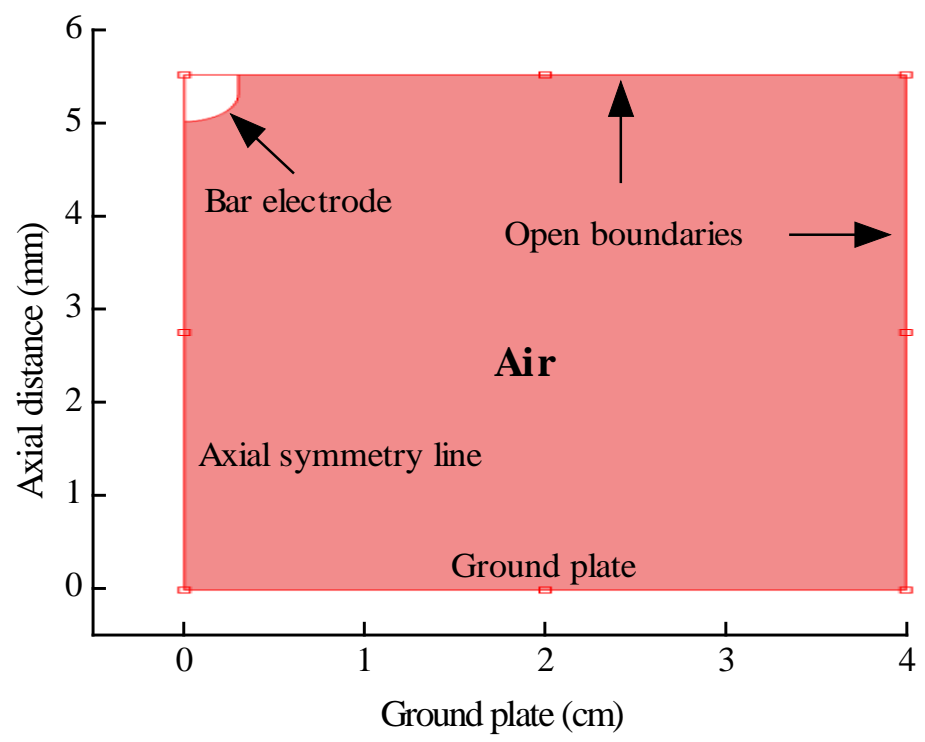

Fig. 1 Computational domain

The bar-plate electrode system model is simplified into a two-dimensional representation using the rotational symmetry. There are two electrodes that separate from each other with a gap of $L=5$ $\mathrm{mm}$, the plate electrode is grounded and the bar electrode is driven with DC $3 \mathrm{kV}$. In this paper, the pressure in air discharge is fixed at $1 \mathrm{~atm}$, and the gas temperature is assumed to be a constant (300 K). Figure 1 shows the computational domain.

\section{Results and Discussion}

Figure 2 shows the discharge current waveform in the electrode gap. The pulse of the discharge current is characterized by a fast rising front and a slower decaying. Some oscillations are found on the decaying curve. The maximum discharge current is higher than $90 \mathrm{uA}$. The width of initial pulse is around $5 \mathrm{~ns}$, and the tail lasts approximately $30 \mathrm{~ns}$. The long tail discharge current waveform may stem from the artificial numerical diffusion.

Figure 3 shows the electron densities at four different times along the axis. A large number of electrons are accumulated in the anode sheath region. The electron density is largest near the anode sheath region and is reduced towards the cathode. Electron distribution area extends to the cathode with the time increases. At time $t=29 \mathrm{~ns}$, in the cathode sheath region, a peak electron density appears due to the secondary electron emission at the cathode surface, which is caused by the effect of secondary electron emission and the ionization dominant in the cathode sheath due to the high electric 
field. Figure 4 illustrates the development of the electron densities visually.

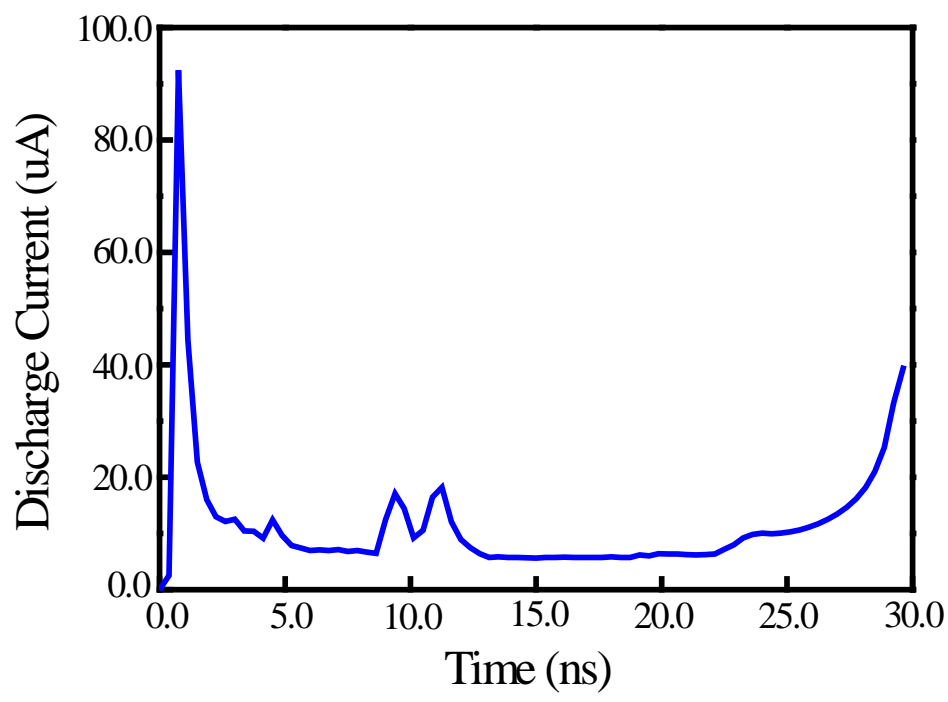

Fig. 2 The discharge current waveform in the electrode gap

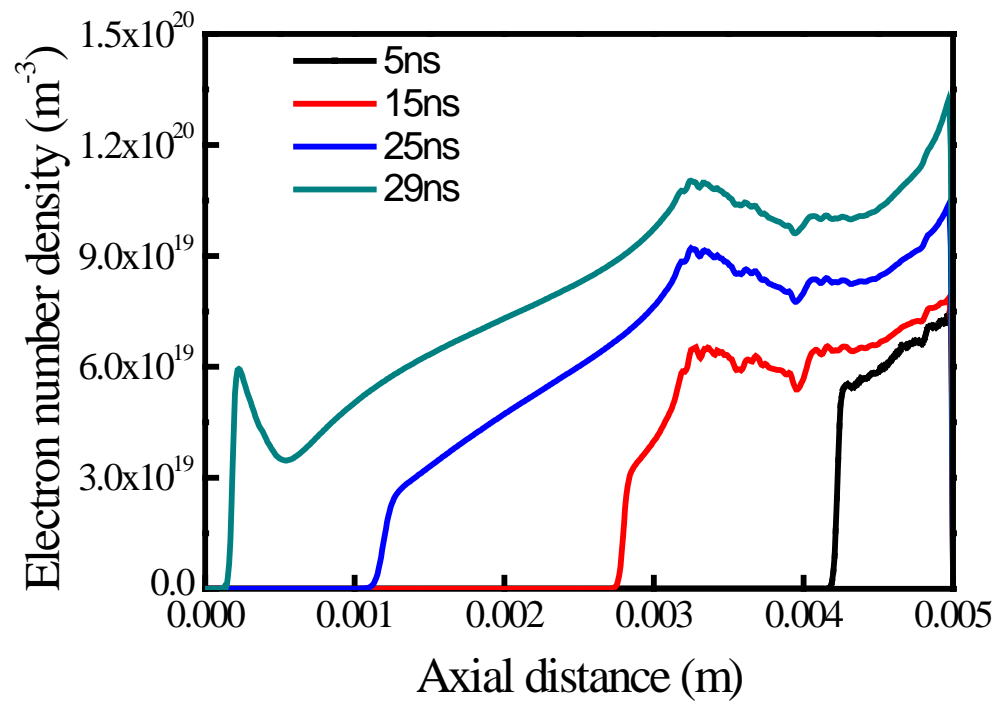

Fig. 3 Two or more references

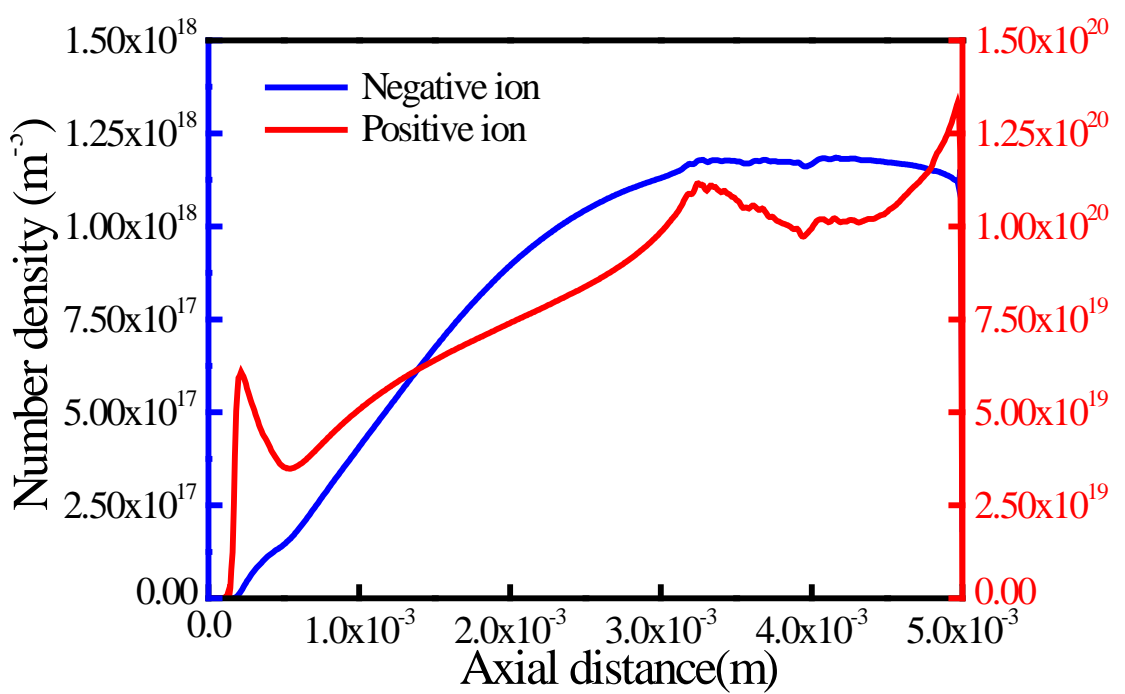

Fig. 4 Positive and negative ion densities along the axis 
Figure 4 demonstrates the plots of positive and negative ion densities along the axis. Similarly to the electron density, the positive ion density follows the same pattern. Close to the anode in the anode sheath region, the positive ion density increases to its maximum value owing to the accumulation of positive charge there. In the cathode sheath region, the positive ion density increases slightly, because the ionization collision caused by the emitted electrons form the cathode produces slow positive ions trying to escape from cathode. We found that negative ion density increases to its maximum value from cathode to anode, while compared to positive ion densities, it is less about 2 orders of magnitude. Therefore, the influence of the negative ion on discharge is relatively small.

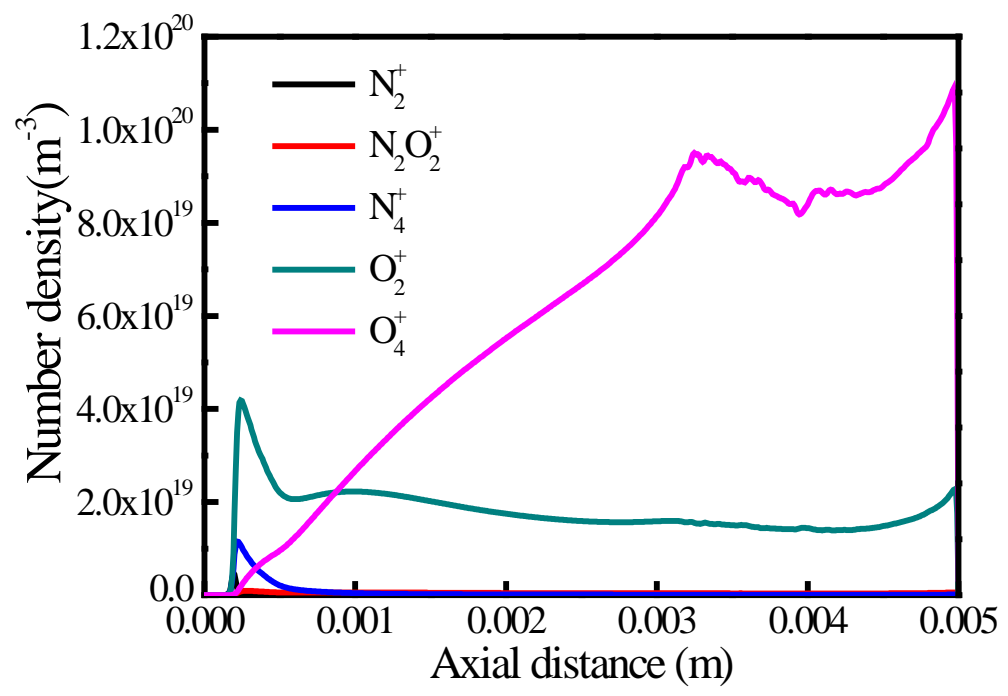

Fig. 5 Positive and negative ion densities along the axis

Figure 5 shows that the positive ions $\mathrm{O}_{4}{ }^{+}$and $\mathrm{O}_{2}{ }^{+}$are dominant compared to the other charged heavy species. $\mathrm{O}_{4}{ }^{+}$as the most number of positive ions is similarly to the electron density near the anode sheath region. It is interesting to note that $\mathrm{N}_{2}{ }^{+}$and $\mathrm{O}_{2}{ }^{+}$sharply increase to the maximum value in the anode sheath region, and keep almost a uniform value in the bulk plasma compared with the distributions of electron and positive ion densities. We also found $\mathrm{O}_{2}{ }^{+}$density significantly greater than $\mathrm{N}_{2}{ }^{+}$because the ionization threshold energy of $\mathrm{O}_{2}(12.06 \mathrm{eV})$ is relatively small compared to the ionization reaction of $\mathrm{N}_{2}(15.6 \mathrm{eV})$. As a consequence of the dominance of $\mathrm{O}_{2}{ }^{+}$and $\mathrm{N}_{2}{ }^{+}$in the cathode sheath region, the air plasma hybrid model is most sensitive to the secondary electron emission coefficient of $\mathrm{O}_{2}{ }^{+}$and $\mathrm{N}_{2}{ }^{+} \cdot \mathrm{N}_{2} \mathrm{O}_{2}{ }^{+}$and $\mathrm{N}_{4}{ }^{+}$are negligible compared to the other ionized species in the air discharge.

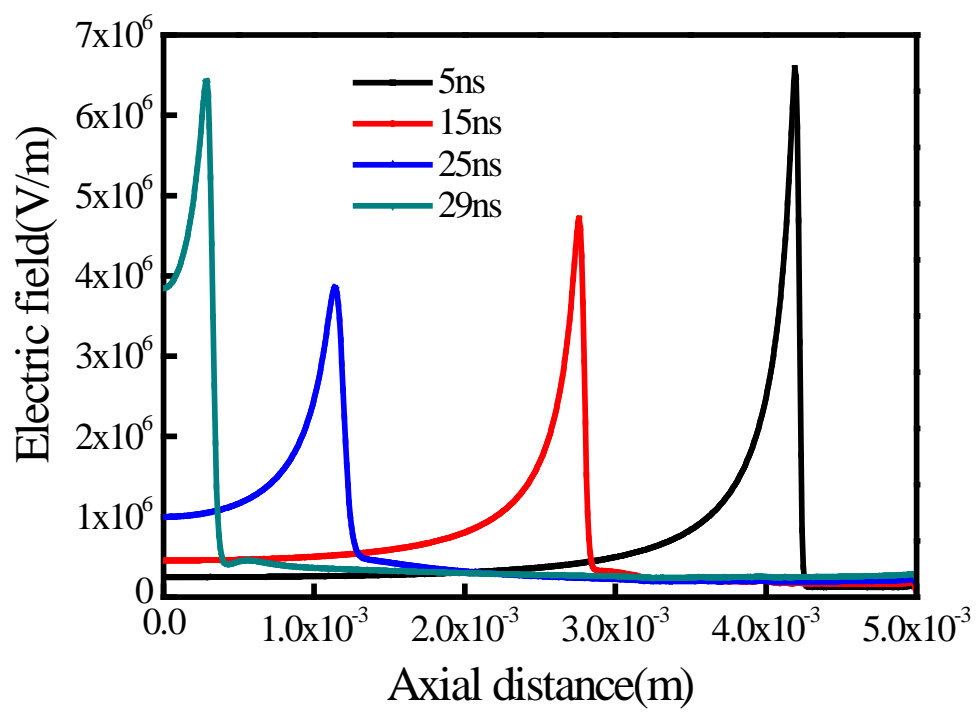

Fig. 6 Distributions of electric field at 4 different time along the axis 


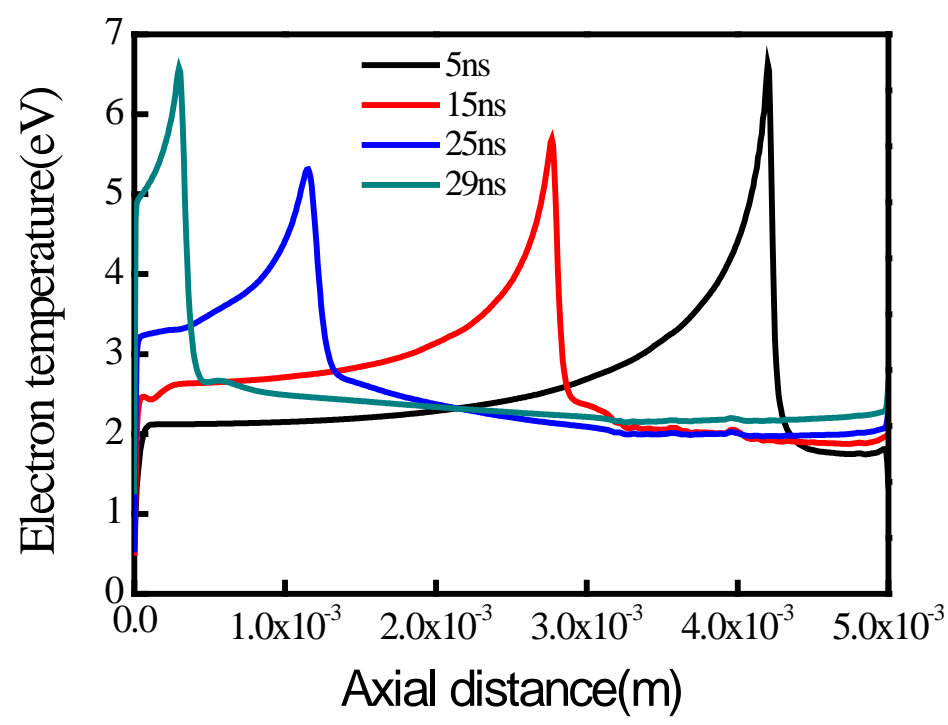

Fig. 7 Electron temperature distributions at 4 different time along the axis

The electron temperature in the air corona discharge is extremely important. Figure 7 reports the electron temperature distributions at different time along the axis in the air discharge. Compared with Figure 6, they have the same pattern. The maximum electron temperature moves toward the cathode. In high electric field, the high electron temperature is the result of Joule heating, which leads to the production of energetic electrons in the cathode sheath region. The electron temperature decreases to a much smaller value and keeps relativelly uniform electron energy in the bulk plasma, which is caused by the electron energy loss incurred from the collision reactions of the electrons with neutral species. The spatial variation of the electron temperature mirrors that of the collision ionization reaction coefficient as well as the speed of energy transfer. Both the results suggest that the secondary electrons are rapidly accelerated in the cathode fall region and the space charge distort the electron field. Thus, the electron temperature in the cathode sheath region is strengthened.

\section{Conclusion}

Numerical simulation offers a better understanding of the physical characteristics of the air corona discharge. In this paper, the numerical simulation of DC air discharge under atmosphere environment is performed using a two-dimensional axisymmetric developed kinetics model that introduced the photoionization effect. The plasma model utilized 12 species and 27 reactions which are based on a self-consistent, multi-component, and continuum description of the air discharge. The important conclusions arrived at based on the present investigations are for the followings:

1) The pulse of the discharge current is characterized by a fast rising front and a slower decaying curve, which includes some oscillations.

2) The electron density is maximum in the anode sheath region and is reduced towards the cathode, electron distribution area extends to the cathode with the time progess, a peak electron density appears due to the secondary electron emission at the cathode surface, which is caused by the effect of secondary electron emission.

3) $\mathrm{O}_{4}{ }^{+}$is dominant compared to the other charged heavy species, $\mathrm{O}_{2}{ }^{+}$and $\mathrm{N}_{2}{ }^{+}$play the key role in secondary electron emission. compared to the other positive ion species, $\mathrm{N}_{2} \mathrm{O}_{2}{ }^{+}, \mathrm{N}_{4}{ }^{+}$are negligible, negative ion and neutral particles play a negligible role in discharge process.

4) Distributions of electron temperature and electric field have the same pattern, and extend along the cathode surface as time progresses. The high electron temperature is the result of Joule heating in the high electric field, while the peak of electric field is caused by applied voltage and distribution of space charge.

The presented hybrid model can contribute to understand the main physical mechanisms and characteristics of the atmospheric pressure air corona discharge, meanwhile, it suggests ways to 
enhancing the electrical insulation performance associated with the high-voltage equipments.

\section{References}

[1] Mahadevan S, Raja L L. 2010. Simulations of direct-current air glow discharge at pressures $~ 1$ Torr: Discharge model validation, Journal of Applied Physics, Vol. 107, No. 9, pp. 093304, 2010.

[2] Macheret S, Shneider M, Miles R. 2002. Nonequilibrium magnetohydrodynamic control of scramjet inliets, 33rd Plasmadynamics and Lasers Conference.

[3] Pancheshnyi S V, Starikovskii A Y. 2003. Two-dimensional numerical modelling of the cathode-directed streamer development in a long gap at high voltage, Journal of Physics D Applied Physics, Vol. 36, No. 21.

[4] Morrow R. 1999. The theory of positive glow corona, Journal of Physics D Applied Physics, Vol. 30, No. 22.

[5] Gordiets B F, Ferreira C M, Guerra V L, et al. 1995. Kinetic modle of a low-pressure $\mathrm{N}_{2}-\mathrm{O}_{2}$ fowing glow discharge”, IEEE Transactions on Plasma Science, Vol. 30, No.4.

[6] Neufeld P D, Janzen A R, Aziz R A. 1972. Empirical equationns to calculate 16 of the transport collision integrals $\Omega(\mathrm{l}, \mathrm{s}) *$ for the lennard-jones (12-6) potential”, Journal of Chemical Physics, Vol. 57, No.3. 